\title{
In situ characterization of transition metal sulfide catalysts by IR probe molecules adsorption and model reactions.
}

\author{
G. Berhault ${ }^{1}$, M. Lacroix ${ }^{1}$, M. Breysse ${ }^{2}$, F. Mauge ${ }^{3}$ and J-C. Lavalley ${ }^{3}$. \\ ${ }^{1}$ Institut de Recherches sur la Catalyse, CNRS UPR 5401, 2, Avenue Albert Einstein, 69626 \\ Villeurbanne Cedex, France. \\ 2 Laboratoire de Réactivité de Surface, CNRS UMR 7609, Université Pierre et Marie Curie, 4, \\ place Jussieu, Casier 178, 75252 Paris Cedex 05, France. \\ ${ }^{3}$ Laboratoire Catalyse et Spectrochimie, CNRS UMR 6506, ISMRA-Université, 6, Boulevard \\ du Maréchal Juin, 14050 Caen Cedex, France.
}

This work reports a detailed characterization of reduced states of $\mathrm{RuS}_{2} / \mathrm{SiO}_{2}$ catalyst by combining catalytic activity measurements and IR probe molecule adsorption. Depending on the solid composition monitored by a progressive reduction these surfaces gradually moves from an acid-base character to a metallic one. Both Lewis and Brønsted acidic sites are created in mild reduction conditions and the Lewis acidic sites play an important role in the activation of sulfur containing molecules and subsequently on their transformations. The hydrogenation properties are related to Ru sites with a low sulfur coordination.

\section{INTRODUCTION}

Transition Metal Sulfides are efficient materials for catalyzing several reactions such as the $\mathrm{C}-\mathrm{X}(\mathrm{X}=\mathrm{S}, \mathrm{N}, \mathrm{O}$, Metal) bond hydrogenolysis, hydrogenation, the selective transformation of organic disulfides into the corresponding thiols as well as the aromatization of cyclic thioethers and the selective ketones amination. Among these solids, $\mathrm{RuS}_{2}$ is one of the most active TMS [1-2]. This indicate that its surface is flexible enough to adapt the proper configuration site required to catalyze this large variety of reactions which demand different intrinsic properties. Theoretical and experimental studies have ascribed the high activities of $\mathrm{RuS}_{2}$, as for $\mathrm{Rh}_{2} \mathrm{~S}_{3}$ and $\mathrm{PtS}_{\mathrm{x}}$ to their weak metal-sulfur bond energy [3]. This property is propitious to the formation of a large number of coordinatively unsaturated sites (CUS) whose properties may be regarded as a Lewis-type center interacting with electron-donating organic substrates [4]. Beside this CUS, the surfaces of TMS also contains some sulfur anions and $\mathrm{SH}$ groups which simultaneously co-exist depending on the nature and on the composition of the surrounding atmosphere. However, there is a lack of characterization of the acid-base properties of these surfaces which are supposed to play an important role in the successive elementary steps involved in the above mentioned reactions. Besides this acidic-base character, Moraweck et al have demonstrated that for small $\mathrm{RuS}_{2}$ clusters encaged into a $\mathrm{Y}$ zeolite, some metallic Ru microdomains may co-exist at the surface of the sulfided particles leading to a metal-sulfide type interface [5]. Accordingly, the surface of a $\mathrm{RuS}_{2}$ particle may 
behave as a metal or as an acid-base material depending on reaction conditions in agreement with the highly reducible character of such a sulfide. The aim of this work was to develop the required tools for characterizing the modification of the surface properties of a silica supported $\mathrm{RuS}_{2}$ induced by a progressive reduction. For this purpose, we used a silica supported $\mathrm{RuS}_{2}$ catalyst as model system because silica is relatively neutral and does not interact too much with the supported sulfide phase. Solid characterizations were performed by combining catalytic measurements with in situ probe molecule adsorption $\left(\mathrm{CH}_{3} \mathrm{SH}, \mathrm{CO}\right.$, pyridine and lutidine). Pyridine was used to detect the Lewis acidity while lutidine was preferred for dosing the Brønsted acidity because of its higher basicity and $\mathrm{CO}$ was selected because its wavenumber is sensitive to the CUS environment. The catalytic properties were determined in two model reactions suspected to reflect different surface properties i.e. the 1butene hydrogenation and the condensation of $\mathrm{CH}_{3} \mathrm{SH}$ into $\mathrm{CH}_{3} \mathrm{SCH}_{3}$.

\section{EXPERIMENTAL}

\subsection{Catalyst preparation}

The silica-supported $\mathrm{RuS}_{2} / \mathrm{SiO}_{2}$ was prepared by the pore filling method using $\mathrm{RuCl}_{3}$ aqueous solutions. The impregnated and dried solid was sulfided at $673 \mathrm{~K}$ with a $15 \% \mathrm{H}_{2} \mathrm{~S}$ $85 \% \mathrm{~N}_{2}$ mixture. After this activation procedure, the solids were cooled down to room temperature in the presence of the sulfur-containing atmosphere, flushed with an oxygen free nitrogen flow and stored in sealed bottles. The Ru loading was 7.5 weight $\%$, the S content corresponds to $\mathrm{RuS}_{2.7}$ and the residual chlorine content was lower than $0.1 \%$.

\subsection{Catalyst reduction and catalytic properties}

These experiments were performed in situ in the same flow microreactor equipped with two parallel detectors, a Flame Photometric Detector (FPD) and a Flame Ionization Detector (FID) in order to detect respectively $\mathrm{H}_{2} \mathrm{~S}$ and the hydrocarbons. The $\mathrm{H}_{2} \mathrm{~S}$ released upon hydrogen reduction was quantified by calibrating the detector with a known concentration of $\mathrm{H}_{2} \mathrm{~S}$ (573 ppm) diluted in hydrogen. The degree of reduction $\alpha$ was defined by the ratio of the amount of $\mathrm{H}_{2} \mathrm{~S}$ eliminated from the solid to the total sulfur content. The reduced catalysts were then contacted at $273 \mathrm{~K}$ with a mixture of $\mathrm{H}_{2}(93.4 \%)$-1-butene(6.6\%) or at $473 \mathrm{~K}$ $\mathrm{N}_{2}(94.4 \%)-\mathrm{CH}_{3} \mathrm{SH}(5.6 \%)$. For both reactions, conversions were kept lower than $10 \%$ in order to avoid mass transfer limitations.

\subsection{FTIR spectroscopy}

FTIR characterization was performed using self-supporting discs of pressed samples. The catalysts were resulfided in situ in the infrared transmission cell according to the procedure already described[4]. Solid reduction was performed with 200 Torr of hydrogen at various temperatures. Several reduction-evacuation cycles were done in order to remove the $\mathrm{H}_{2} \mathrm{~S}$ formed upon reduction. Then, the samples were evacuated at $393 \mathrm{~K}$ for $30 \mathrm{~min}$ prior to molecule adsorption. Probe molecule adsorptions were performed at $100 \mathrm{~K}$ for $\mathrm{CO}$ or at room temperature for the others probes. The reduced catalysts were contacted with 1 Torr of $\mathrm{CO}, 2$ Torr of pyridine (Py) and 2-6 dimethylpyridine (DMP) or 4 Torr of $\mathrm{CH}_{3} \mathrm{SH}$ and then evacuated. The IR spectra were recorded using a Nicolet 60SX FTIR spectrometer. Band intensities were corrected from slight differences in catalyst weight and adjusted to $10 \mathrm{mg}$. 


\section{RESULTS}

\subsection{Solid reduction and catalytic properties}

The starting point of this work was to examine how the $\mathrm{RuS}_{2} / \mathrm{SiO}_{2}$ catalyst behaves towards a hydrogen treatment. Preliminary TPR experiments have evidenced that the silica support sulfided in the same experimental conditions does not retained any detectable amount of $\mathrm{H}_{2} \mathrm{~S}$. It was also observed that over the $\mathrm{RuS}_{2} / \mathrm{SiO}_{2}, \mathrm{H}_{2} \mathrm{~S}$ is mostly removed upon heating and then the solid rapidly equilibrates when treated in isothermal conditions. Accordingly all solids were reduced at the desired temperature and left in isothermal conditions for only $2 \mathrm{~h}$

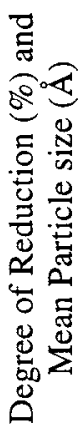

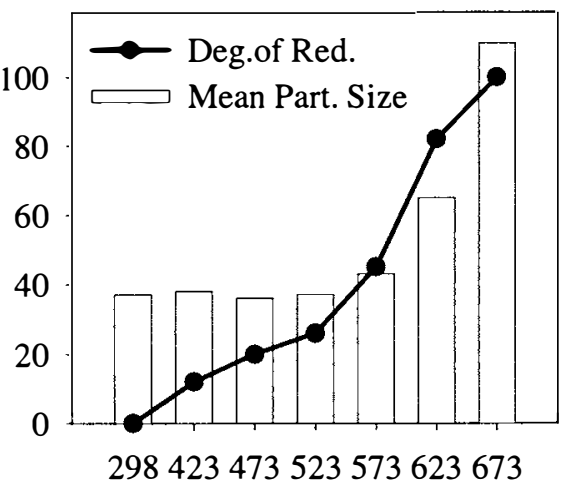

Reduction Temperature $(\mathrm{K})$

Fig. 1. Evolution of the degree of reduction and of the mean particle size as a function of the temperature of reduction.

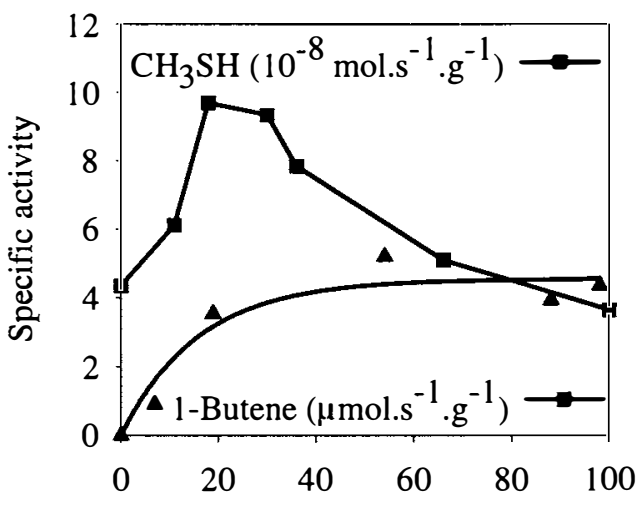

Degree of Reduction (\%)

Fig. 2. Evolution of the catalytic properties as a function of the degree of reduction

Figure 1 shows the evolution of the degree of reduction versus the reduction temperature. At $673 \mathrm{~K}$ the amount of $\mathrm{H}_{2} \mathrm{~S}$ released from the solid corresponds to that determined by chemical analysis indicating that the solid is entirely reduced. This figure also reports the mean particle size determined by HREM. The non-reduced solid could be considered as an assembling of spherical particle with a mean diameter of circa $35 \AA$ with a narrow distribution since the standard deviation was about $8 \AA$. Neither the particle size nor the distribution width were affected up to a reduction temperature of $573 \mathrm{~K}$. At $623 \mathrm{~K}$ both parameters increase and the XRD patterns reveal the concomitant presence of the $\mathrm{RuS}_{2}$ and the metal Ru phases while only the latter is detected when the solid is reduced at $673 \mathrm{~K}$. These data indicate that the pyrite phase preserves its morphology up to a reduction temperature of $573 \mathrm{~K}$.

Figure 2 shows the variation of the catalytic activities as a function of the degree of reduction. The non-reduced solid is already active for the condensation reaction. As far as sulfur is removed from the catalyst the activity increases, reaches a maximum for $\alpha=20 \%$ and then continuously decreases for further sulfur removal. By contrast, the butane formation follows a distinct trend i.e. the non-reduced solid is inactive and the activity increases up to $\alpha$ $=40 \%$ and then stabilizes. The different comportment of the catalyst towards both reactions strongly suggest that they require different type of sites. 


\subsection{Pyridine (Py) and 2-6 dimethylpyridine (DMP) adsorption.}

Py interaction with the silica support gives rise to several bands characterizing Lewis and Brønsted sites. However, this interaction is weak because a low signal is detected after desorption at $423 \mathrm{~K}$ (Fig. 3). By contrast, these bands remain on the Ru catalyst. The spectra recorded on the non-reduced sample (NR) exhibits intense bands at 1602 and $1444 \mathrm{~cm}^{-1}$ involving Lewis (L) acidity as well as some weaker ones in the range 1500-1580 and 1610$1660 \mathrm{~cm}^{-1}$ characterizing the Brønsted (B) sites while the band at $1485 \mathrm{~cm}^{-1}$ arises from both $\mathrm{L}$ and $\mathrm{B}$ sites. Solid reduction up to $473 \mathrm{~K}$ brings about an increase of band intensities without any change in their positions suggesting an increase in the number of acidic sites without a large modification of their strength. The diminution of band intensities for a reduction temperature of $573 \mathrm{~K}$ is rather surprising since the amount of $\mathrm{H}_{2} \mathrm{~S}$ removed has drastically increased. This unexpected behavior suggests a strong modification of the surface properties because particule size remains unaffected.

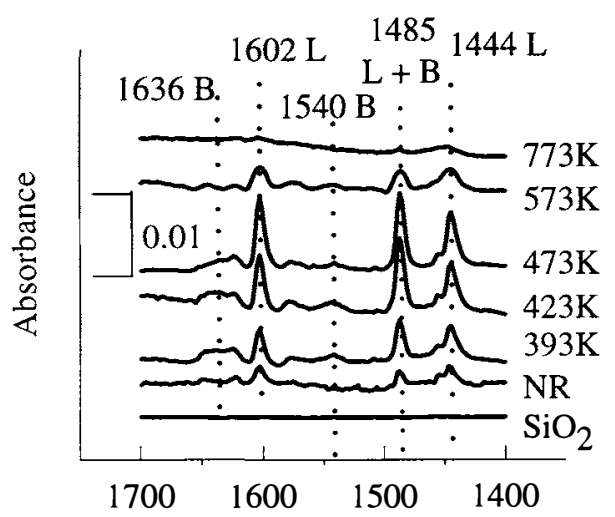

Wavenumbers $\left(\mathrm{cm}^{-1}\right)$

Fig. 3. IR spectra of adsorbed pyridine for various reduced $\mathrm{Ru}$ catalyst.

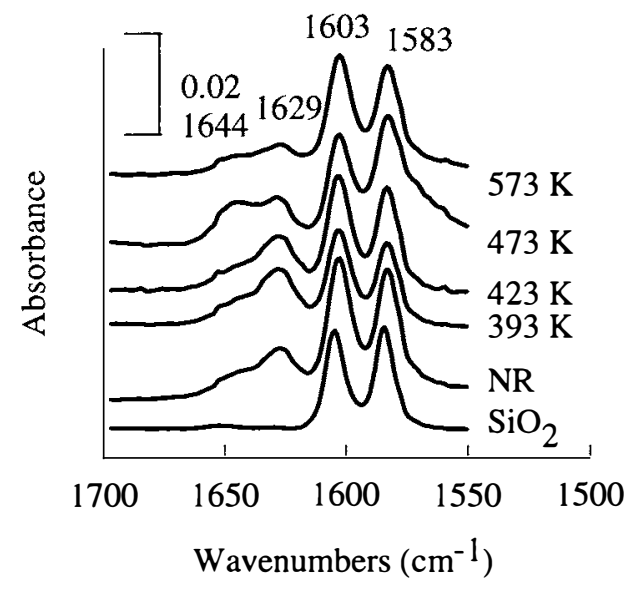

Fig. 4. IR spectra of adsorbed DMP for various reduced $\mathrm{Ru}$ catalyst.

As evidenced in Fig. 3 solid reduction also modifies the concentration of B sites. However the band related to pyridinium ion is too weak for estimating the variation of their concentration. Figure 4 shows, the IR spectra of adsorbed DMP recorded after an evacuation at RT. In the 1550-1700 $\mathrm{cm}^{-1}$ range, four main bands are detected. The most intense ones located at 1583 and $1603 \mathrm{~cm}^{-1}$, whose intensities vary little with the pretreatment, correspond to DMP adsorbed on both L and B sites. The two others at 1629 and $1644 \mathrm{~cm}^{-1}$ agree fairly well with those reported in the literature for protonated species and they may be respectively ascribed to $v 8 \mathrm{~b}$ and $v 8 \mathrm{a}$ vibration modes of $\mathrm{DMPH}^{+}$species [6]. These bands are not present on the silica support treated in the same conditions and their intensities slightly varies with the solid reduction and a maximum emerges at a reduction temperature of about 423 and $473 \mathrm{~K}$. 


\section{3. $\mathrm{CH}_{3} \mathrm{SH}$ and $\mathrm{CO}$ adsorption}

Adsorption of $\mathrm{CH}_{3} \mathrm{SH}$ on silica leads to four IR bands located at 3012, 2948, 2858 and $2590 \mathrm{~cm}^{-1}$ which corresponds respectively to the $\mathrm{va}\left(\mathrm{CH}_{3}\right), v \mathrm{~s}\left(\mathrm{CH}_{3}\right), 2 \delta \mathrm{d}\left(\mathrm{CH}_{3}\right)$ and to the $v(\mathrm{SH})$ stretching mode of the free $\mathrm{CH}_{3} \mathrm{SH}$ molecule. The presence of the latter suggests that the probe is only physisorbed on the support. This assumption is confirmed by the flat spectrum observed after evacuation at RT (Fig. 5).

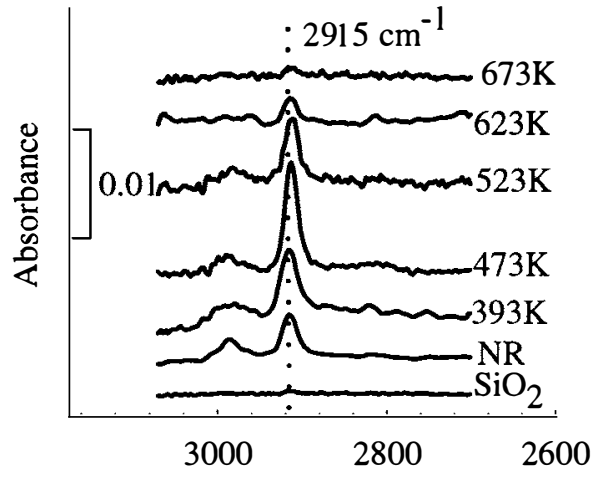

Wavenumbers $\left(\mathrm{cm}^{-1}\right)$

Fig. 5. IR spectra of adsorbed $\mathrm{CH}_{3} \mathrm{SH}$ for various reduction temperatures (Evacuation at RT). NR : non-reduced solid

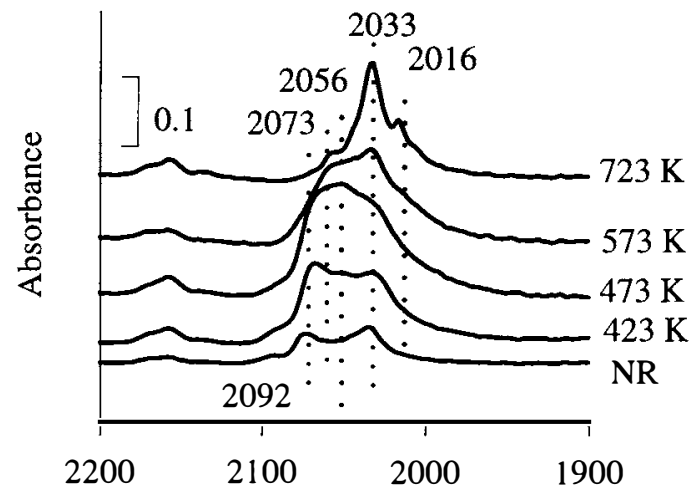

Wavenumbers $\left(\mathrm{cm}^{-1}\right)$

Fig. 6. CO adsorption spectra for various reduction temperatures (Evacuation at RT). NR : non-reduced solid

On the $\mathrm{Ru}$ catalysts the $\mathrm{va}\left(\mathrm{CH}_{3}\right)$ bands are shifted towards lower wavenumbers and the $v(\mathrm{SH})$ stretching band was no longer observed. Band intensities slightly decrease upon evacuation and these bands are still detected after evacuation at 393 or $473 \mathrm{~K}$. These data suggest that $\mathrm{CH}_{3} \mathrm{SH}$ heterolytically dissociates leading to the formation of thiolate species linked to a Lewis type center whose IR signature corresponds to the intense band observed at $2915 \mathrm{~cm}^{-1}$. The amount of such a species increases upon reduction and its concentration exhibits a maximum for $\operatorname{Tr}=473 \mathrm{~K}$ in agreement with the evolution of $\mathrm{L}$ sites previously determined using Py as probe molecule.

$\mathrm{CO}$ adsorption leads to several bands and a shift of band positions towards lower wavenumber appears when the reductive treatment becomes more severe (Fig.6). This is in favor of a progressive sulfur depletion around $\mathrm{Ru}$. By comparison with literature data, the band at 2093 $\mathrm{cm}^{-1}$ indicates the presence of CUS in a highly sulfided environment while part of the band at $2033 \mathrm{~cm}^{-1}$ with the band at $2016 \mathrm{~cm}^{-1}$ could be related to the formation metallic $\mathrm{Ru}$ [7]. The bands at 2073, 2056 and part of that at $2033 \mathrm{~cm}^{-1}$ characterized Ru cations in a lower sulfur coordination.

\subsection{Relationship to catalytic activities}

Figure 7 shows the evolution of the intensities of the thiolate and Py-L bands with the degree of reduction follows the same trend that the condensation reaction suggesting an adsorption of the $\mathrm{S}$ containing molecules on an electron accepting site. The similar behavior of the intensity of the high frequency $\mathrm{CO}$ bands indicates that these $\mathrm{Ru}$ sites are still in a rich 
sulfur environment. The smoother variation in the relative amount of Brønsted sites and the lower temperature at which the maximum is observed shows that these sites do not play an important role in this reaction.

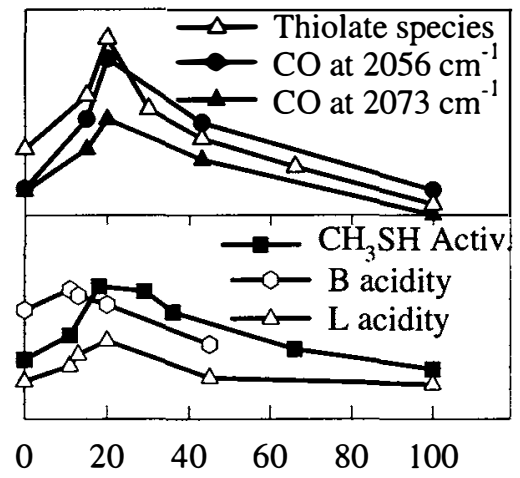

Degree of Reduction (\%)

Fig. 7. $\mathrm{IR}$ data and $\mathrm{CH}_{3} \mathrm{SH}$ activity relationship

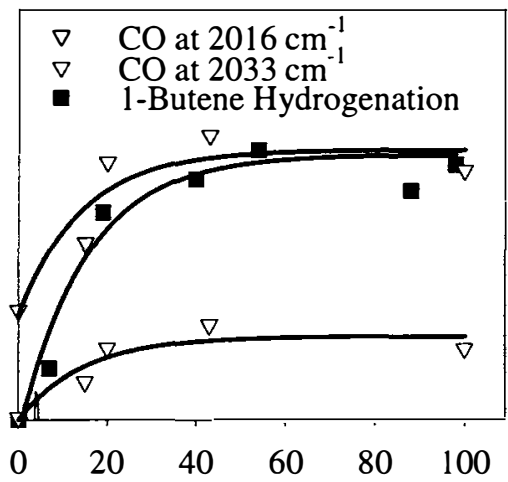

Degree of Reduction (\%)

Fig. 8. IR data and hydrogenation activity relationship

By contrast, highly depleted $\mathrm{Ru}$ sites are required to performed the hydrogenation reaction as shown in Fig. 8. Indeed, a nice correlation was found between the 1-butene hydrogenation activity and the low $\mathrm{CO}$ bands.

\section{CONCLUSIONS}

These data evidence that the properties of the $\mathrm{RuS}_{2}$ surface progressively moved from an acid-base character to a metallic one. The properties of the several surface species whose concentration and nature are strongly dependent on the sulfur to metal ratio have been characterized by means of IR spectroscopy and model molecules conversion. The modification of the surface properties of transition metal sulfide catalysts as a function of the surrounding atmosphere and particularly of the relative $\mathrm{H}_{2}-\mathrm{H}_{2} \mathrm{~S}$ partial pressure should be taken into account when these solids are used in complex reaction such as dibenzothiophene conversion. This approach developed on a model catalyst can be now applied to more complex catalytic systems.

\section{REFERENCES}

1. T.A. Pecoraro and R.R. Chianelli, J. Catal., 67 (1981) 430.

2. M. Lacroix, N. Boutarfa, C. Guillard, M. Vrinat and M. Breysse, J. Catal., 120 (1989) 473.

3. J.K. Nørskov, B. S. Clausen and H. Topsøe, Catal. Lett., 13 (1992) 1.

4. G. Berhault, M. Lacroix, M. Breysse, F. Maugé, J.C. Lavalley, H. Nie and L. Qu, J. Catal., 178 (1998) 555.

5. B. Moraweck, G. Bergeret, M. Cattenot, V. Kougionas, C. Geantet, J.L. Portefaix, and M. Breysse, J. Catal., 165 (1997) 45.

6. P.A. Jacobs and C.F. Heylen, J. Catal. 34 (1974) 267.

7. K. Kostov, H. Rauscher, and D. Menzel, Surf. Sci., 278 (1992) 62. 